\title{
Les mycétomes du sud-est de la Bulgarie
}

\author{
par V. A. BALABANOFF \\ Institut scientifique dermatovénérologique de l'Académie de Médecine de Sofia, Bulgarie
}

RESUME. D'après les recherches de l'auteur, la Bulgarie occupe une place importante en Europe par le nombre des cas de mycétomes qui ont été diagnostiqués dans ce pays de climat tempéré d'une superficie relativement petite. Sur les 18 mycétomes ( 8 d'origine fongiques et 10 actinomycosiques) 10 cas, dont 7 fongiques, proviennent du Sud Est du pays, près de la Mer Noire, région voisine de l'Asie Mineure au climat $\mathrm{sec}$ et chaud, ce qui confirme l'influence du climat sur le développement de la maladie. Les 6 mycétomes fongiques à grains noirs ont pour agent Madurella mycetomi, tandis que Monosporium apiospermum est l'agent d'un mycétome à grains blancs. Les agents des mycétomes actinomycosiques sont des Nocardia et Actinomadura madurae. Malgré l'action favorable in vitro de divers antibactériens, y compris le nitroxoline (Nibiol), les mycétomes actinomycosiques sont assez résistants à la thérapeutique et leur évolution est souvent rapide. Un diagnostic précoce s'impose pour éviter l'atteinte osseuse et la nécessité de l'amputation.

\section{Mycetomas originated from South-East Bulgaria.}

SUMMARY. According to investigations by the author, Bulgaria occupies a major position by the number of mycetomas in European countries with temperate climate and small superficies. From 18 cases of mycetomas, 10 cases, whom 7 were maduromycetomas or eumycetomas, originated from South-East Bulgaria, near Black Sea and Asia Minor, with warm and dry climate. This confirms that the climate has a definite influence on the disease. 6 maduromycetomas with black granules were caused by Madurella mycetomi and the only white granules mycetoma was caused by Monosporium apiospermum. Actinomycetomas were caused by Nocardia species and Actinomadura madurae. Despite the favorable effect of antibacterial compound (with special reference to Nibiol/Nitroxolone), in vitro, the actinomycetomas are more resistant to the therapy and they show a more rapid evolution. It is necessary to make a quick and early diagnosis to avoid an invasion of the underlying bones and the necessity of an amputation.

Accepté le 5 février 1980.

Annales de Parasitologie humaine et comparée (Paris), t. 55, $\mathrm{n}^{\circ} 5$ 
Le nombre des cas de mycétomes en Europe s'est précisé au cours des années. Dans l'enquête mondiale publiée en 1967 par F. Mariat (16) répondant à l'envoi d'un questionnaire, 14 cas européens sont indiqués. Desai et coll. (9) en trouvent 45 en 1970. Mais Nicolau et Avram (17), dix ans avant, en avaient signalé 100 cas, dont 12 en Roumanie, 15 en Italie, 9 en Grèce et 11 en U.R.S.S. La monographie publiée par Avram en 1969 mentionne 31 cas roumains dont 25 étudiés par lui. La Roumanie devient ainsi le premier pays européen pour le nombre de mycétomes " une île mycétomique dans les pays de climat tempéré du Nord ». Cependant comme nous allons le voir, la Bulgarie, située immédiatement au Sud, lui fait concurrence pour le nombre des cas de mycétomes, compte tenu de sa superficie moins étendue. Latapi (15) cite les auteurs européens qui ont décrit des mycétomes dans les pays situés au Sud de l'Europe, autour de la Méditerranée. En U.R.S.S., Kaschkin (13) donne les noms des 8 auteurs qui ont étudié des cas de mycétomes dont le nombre est restreint étant donné l'étendue considérable de ce pays. Nous évoluons aujourd'hui le nombre des cas de mycétomes décrits en Europe à plus de 120-150.

En Bulgarie, les trois premiers cas de mycétomes, tous à grains noirs, semblent avoir été décrits par Béron en $1931(7,8)$. Depuis ce nombre s'est accru, en particulier ces 10 dernières années, grâce au perfectionnement des techniques du diagnostic mycologique $(2,3,4,5)$. Nous avons pu en signaler 18 cas et la Bulgarie occupe ainsi la deuxième place par le nombre des cas de mycétomes dans les pays d'Europe. En outre, durant les 6 dernières années, Jekov $(11,12)$ a décrit 12 cas de Nocardiose pulmonaire et 5 cas d'Actinomycose à Actinomyces iraelii dans le seul Institut de Pneumologie de la ville de Pernik; ceci montre l'importance accrue des mycoses et actinomycoses que l'on ne reconnaît pas bien encore et ne recherche pas suffisamment.

\section{Matériel et méthodes}

Sur les 18 cas de mycétomes fongiques ou actinomycosiques, l'auteur en a étudié 13 , à la fois des points de vue clinique, radiologique, histopathologique, mycologique et bactériologique; l'observation directe des grains dans le pus, en sérum physiologique ou dans la potasse à $15-30 \%$ a été pratiquée. Les frottis de pus et grains écrasés ont été colorés par le Gram (modification de Kinioun), les coupes histologiques par la méthode de Gram, par celle de Wesselinov $(3,22)$ et par le P.A.S. La culture des agents de mycétomes a été obtenue en ensemençant les grains, le pus ou des fragments de biopsie sur des tubes de gélose de Sabouraud glucosée à $2 \%$, placés à 24 et $37^{\circ}$. Des cobayes ont également été inoculés.

Ces méthodes permettent de différencier les eumycètes au mycélium tubulaire et septé, des actinomycètes aux filaments fins (inférieurs à $1 \mu$ ) ramifiés, fragmentés chez Nocardia, non fragmentés et Kinioun (21) négatif chez Actinomadura. Une étude physiologique et biochimique suivant Gordon et Mihm (10) est nécessaire pour cer- 
taines espèces d'actinomycètes aérobies. La production de pigment brun diffusible, les études de la lactopositivité et de la température optimum de croissance ont été nécessaires pour identifier les cultures de mycétomes à grains noirs (5). Enfin, la sensibilité envers les antibiotiques et les sulfonamides a été étudiée avec les souches de Nocardia et d'Actinomadura $(4,6)$.

\section{Résultats}

Les 18 cas de mycétome sont présentés dans le tableau $I ; 8$ sont dús à des champignons et 10 à des actinomycètes. Ils ont pour agents :

1. fongiques: Madurella mycetomi (grains noirs) (fig. 1), et Monosporium aspiopermum (grains blancs) (fig. 3);

2. actinomycosiques: Nocardia asteriodes, Actinomadura madurae (fig. 4 et 5), Nocardia sp., Actinomyces transvalensis. Ils correspondent aux agents de mycétomes signalés par Avram en Roumanie: Madurella mycetomi, Monosporium aspiopermum, Nocardia asteroides, etc... (1).

Tous les mycétomes fongiques, à l'exception d'un seul à grains sombres dont l'agent n'a pas été identifié, proviennent du Sud-Est de la Bulgarie (fig. 2) dont les 10 cas sont plus spécialement étudiés ici. Il s'agit de 8 hommes et 2 femmes entre 6 et 67 ans, des campagnards principalement qui marchent pieds nus. Un seul mycétome est localisé à la main, tous les autres cas sont aux pieds et 7 sur 9 au pied gauche. D’ailleurs sur les 18 mycétomes bulgares, 16 sont localisés aux pieds et 13 au pied gauche.

Cette région du Sud-Est de la Bulgarie est située au voisinage de l'Asie Mineure ; son climat chaud et sec est subtropical (14) avec présence d'une riche végétation d'épineux, en particulier d'acacia (19-20). Du district de la ville de Yambol proviennent 3 cas ; de celui de Bourgas, près du littoral Sud de la Mer Noire, 3 autres cas ; enfin 1 cas dans chacun des districts des villes de Aytos, Hoskovo, Pervomay et Stara Zagora.

Une femme âgée (E.P.N. 67 ans, $\mathrm{n}^{\circ} 14$ du tableau I) (fig. 4) présente un intérêt spécial : originaire de la région de Yambol, elle est atteinte d'un mycétome à Actinomadura madurae depuis 11 ans. Il a provoqué des foyers ostéolytiques (géodes) non seulement des métatarsiens et du calcanéum, mais aussi du tibia et du péroné. Elle présente également une infection endothrix du cuir chevelu par Trichophyton violaceum avec onychomycose. La malade qui a refusé l'amputation a été nettement améliorée par une cure de Nitroxoline (Nibiol).

Les souches d'actinomycètes aérobies isolées de mycétomes et de nocardiose pulmonaire sont sensibles in vitro à certains antibiotiques ou antimicrobiens :

1. Biseptol $=2,4$ diamin-5-(3, 4, 5 trimetoxibenzil) pyrimidin et 5-metyl-3-sulanilamid-izoxazol ; 


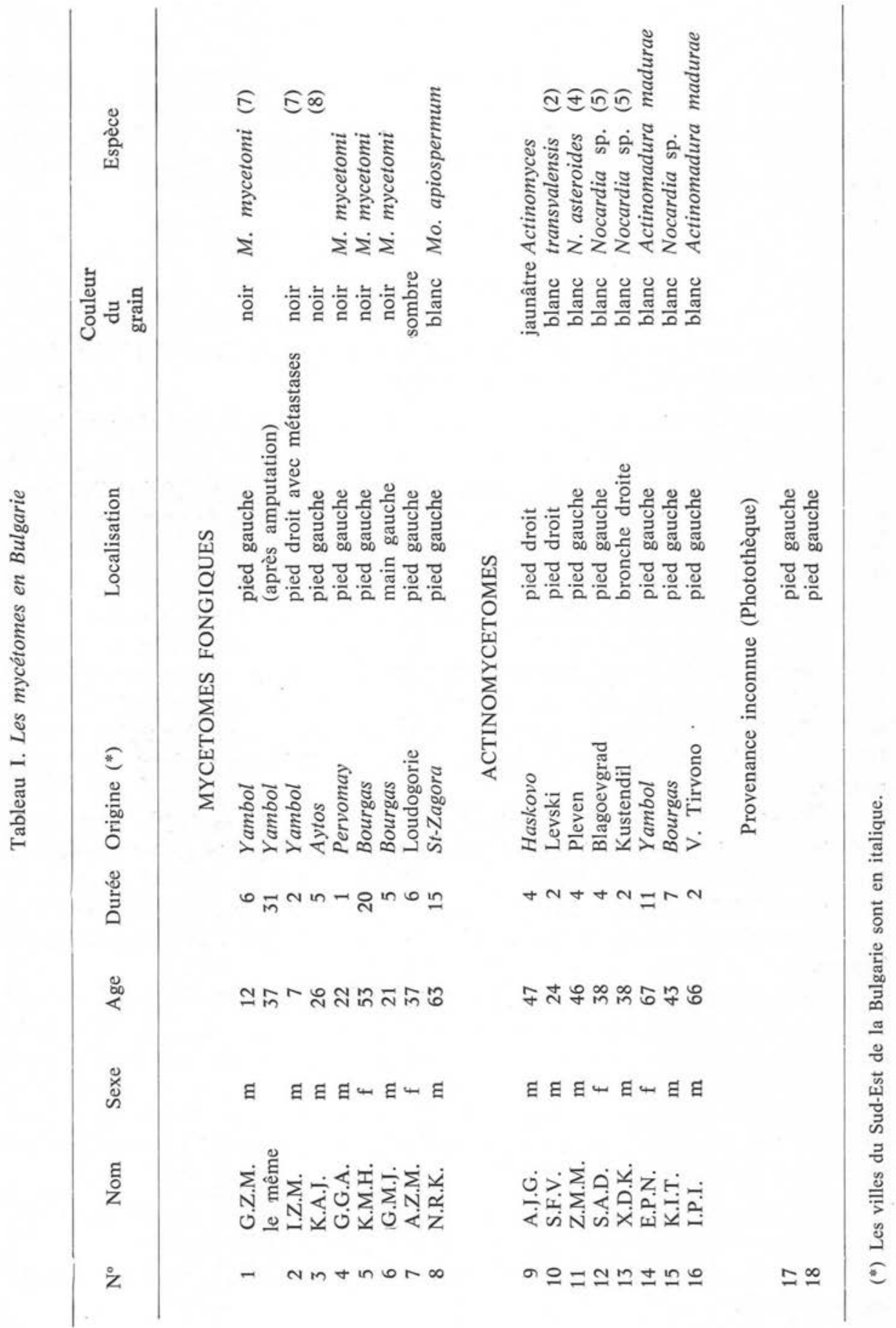



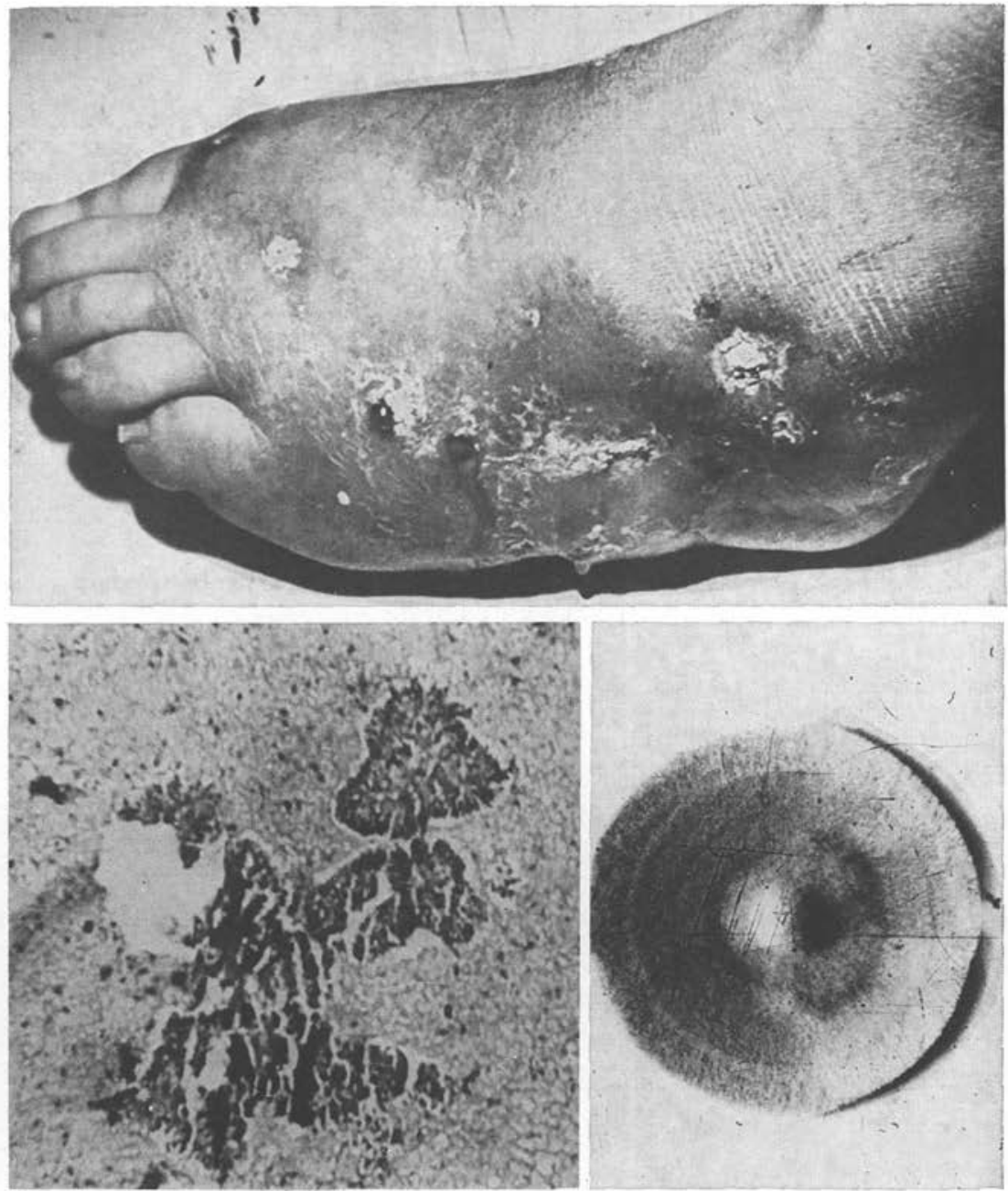

Fig. 1. Maduromycétome à grains noirs du pied gauche $\left(\mathrm{n}^{\circ} 4\right.$ du tableau I). Grain multiloculé, fissuré avec mycélium septé et vésiculé. Culture de Madurella mycetomi (Laveran) Brumpt 1905.

2. Tetraolean $=$ Tetracyclinum hydrochloricum et Oleandomycinum phosphoricum, resp. Triacetyloleandomycinum $(4,0 \mathrm{mcg} / \mathrm{ml})$, etc...

L'activité antiactinomycètes du nitroxoline a été spécialement étudié (tableau $I$ ) ; elle est égale à celle des antibiotiques les plus puissants (6). Cet antimicrobien agit également in vivo, provoquant une amélioration clinique certaine; mais il doit être employé précautionneusement avant atteinte osseuse ou en complément d'intervention chirurgicale. 


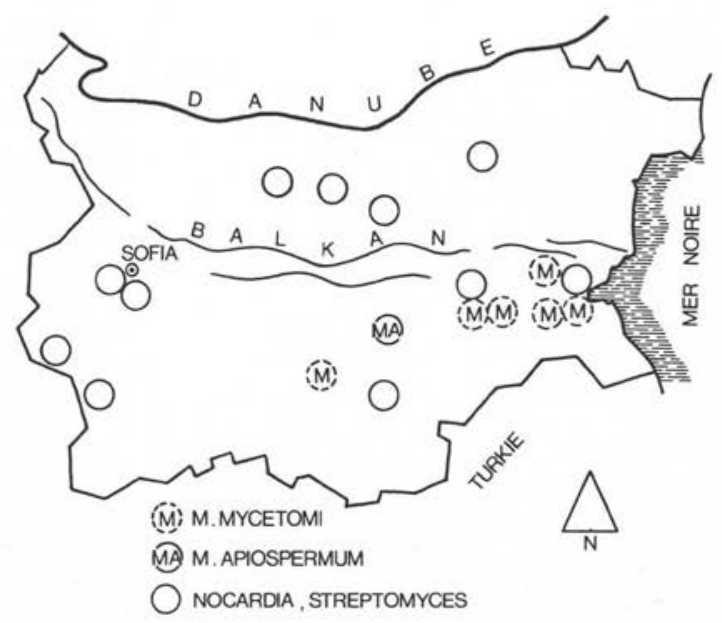

Fig. 2. Répartition géographique des mycétomes en Bulgarie. Les Maduromycétomes occupent le Sud-Est du pays.
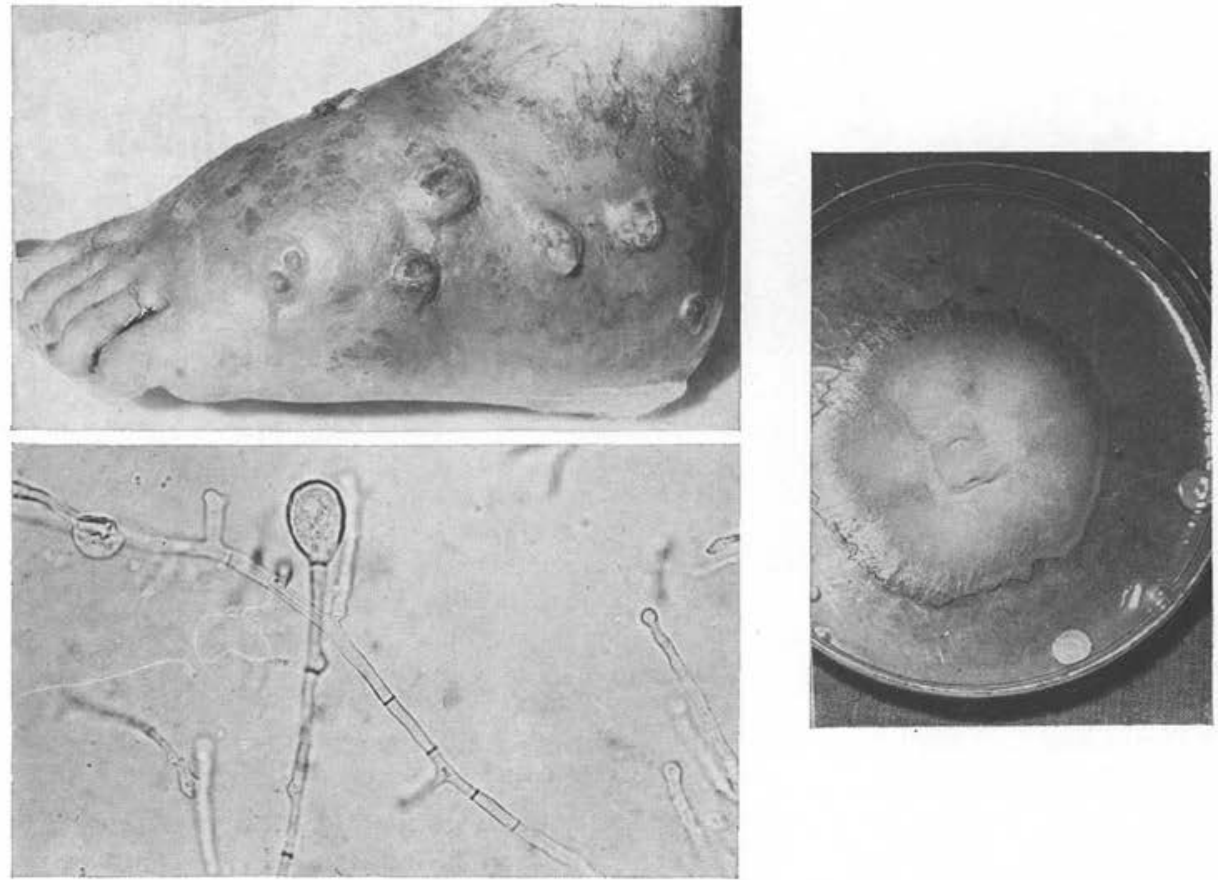

Fig. 3. Maduromycétome à grains blancs par Monosporium apiospermum Saccardo 1911 (Stade imparfait d'Allescheria boydii Shear 1921) ( $\mathrm{n}^{\circ} 8$ du tableau I). Culture sur gélose glucosée de Sabouraud. Spores apicales. 

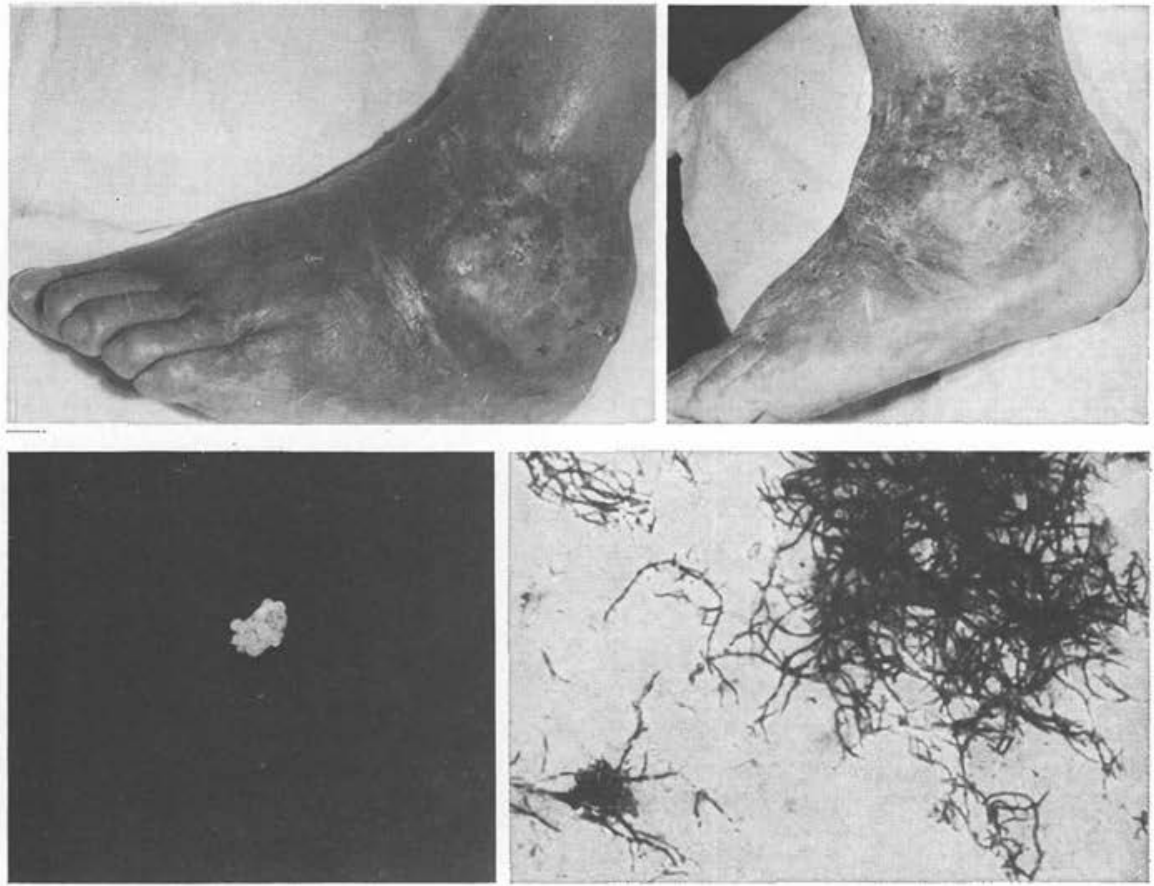

Fig. 4. Actinomycétome par Actinomadura madurae Lechevalier et Lechevalier, 1970 (S. madurae/Vincent/Ochoa-Sandoval, 1951) ( $\mathrm{n}^{\circ} 14$ du tableau I). Lésions avant et après thérapie avec Nitroxoline-Nibiol. Culture sur gélose glucosée de Sabouraud. Micromorphologie: filaments non septés (Gramm).

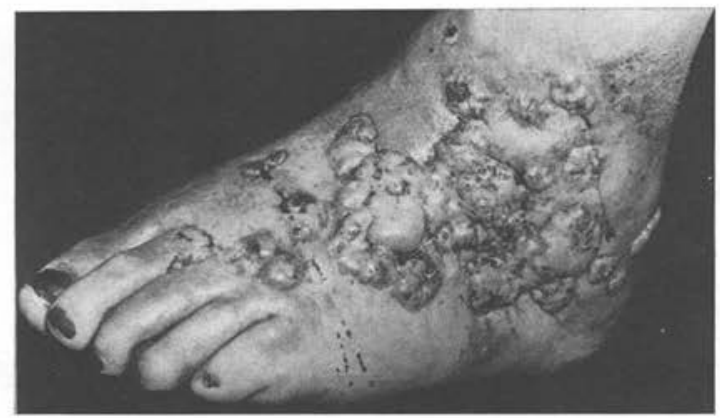

Fig. 5. Actinomycétome par Actinomadura madurae ( $\mathrm{n}^{\circ} 16$ du tableau I). 
Tableau II. Activité antiactinomycotique (actinomycètes aérobies) du Nitroxoline (Nibiol)

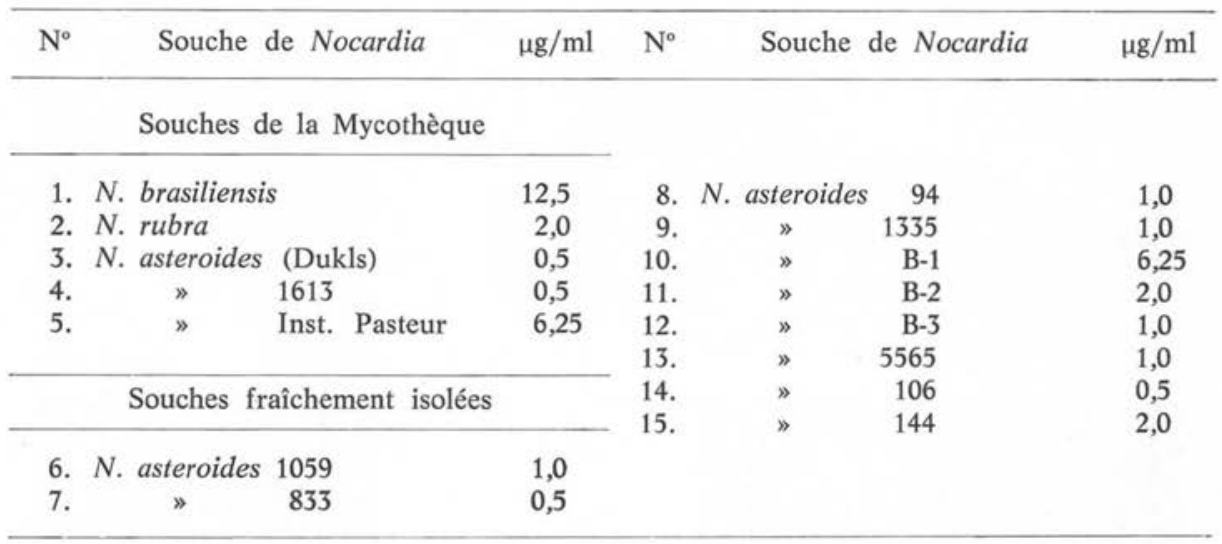

Dans les cas de mycétome, les actinomycètes sont au moins aussi pénétrants et redoutables que les champignons. Ils progressent même plus rapidement et les destructions provoquées en 10 à 30 ans par ces derniers, sont réalisées en 2 à 4 ans par les actinomycètes (4). Une thérapeutique précoce antiactinomycosique s'impose comme complément d'excision ou d'amputation.

Nous avons constaté des erreurs de diagnostic avec la maladie de Kaposi (5), la piodermite, l'ostéomyélite, la tuberculose cutanée...

C'est un plaisir pour moi d'exprimer ma reconnaissance à $\mathrm{M}^{\mathrm{me}} \mathrm{D}$. Bogoslovova pour son assistance technique.

\section{Bibliographie}

1. Avram A.: Micetomele in Romania, 1969, Ed. RSR, Bucuresti.

2. Balabanoff V.A.: Critical survey of medical mycological literature in Bulgaria for the periode 1946-1961. Mycopathol. et Mycol. Appl. 1963, 20, 157-173.

3. Balabanoff V.A.: Uber die Anwendungsmöglichkeiten der Vegetabilien-farbverfahren nach Wesselinoff in der Mycologie und in der Histopathologie. Zbl. Bakter. I Orig., 1960, 178, 135-139.

4. Balabanoff V. A. : Manurafuß durch Nocardia asteroides. Castellania, 1977, 5, 91-94.

5. Balabanoff V.A.: Die Mycetome in Bulgarien und deren Behandlung. Verhandlung der D.D.G. XXXI Tagung in Köln. Supplementum II zu « Der Hautarzt », 1977, 28, 180-181.

6. Balabanoff V.A.: Marinova V., Jekov K.: On the antiactinomycetous activity of 5-Nitrox (Nitroxoline). Proceedings VIII International Congress Infect. Parasit. Dis., Varna 2-6 oct., 1978, 140-143.

7. Beron B. : Zwei autochtone Fälle von Mycetoma mit schwarzer Körner. Derm. Wschr. 1931, 92, 265-272.

8. Beron B.: Dritter Fall von Madurafu $\beta$ in Bulgarieu. Derm. Wschr 1931, 43, 1673.

9. Desai S. C. et al. : Studies on Mycetoma. Ind. J. Surg. 1970, 32, 427-447. 
10. Gordon R. E., Mihm J. M.: The type species of the genus Nocardia. J. Gen. Microbiol., 1962, 27, 1-10.

11. Jekov K. : Etiologic diagnosis of pulmonary actinomycosis and nocardiosis. Epidemiol. microbiol. infect. bol. (Sofia), 1979, 16, 166-171.

12. Jekov K., Torodov D. : A case of Pulmonary Nocardiosis. Pneumol. $i$ ftisiatr. (Sofia), 1976, 13, 150-154.

13. Kaskin P. N. : Medizinskaya mikologia, 1962, Medgiz, Moskwa.

14. Klüicken N.R. et al. : Zur Epidemiologie, Klinik und The rapie der Mycetome in Westafrika. Hautarzt, 1965, 16, 1-16.

15. Latapi F. : Das Mycetom. In J. Jadassohn Hbh f. Haut u. Geschkrkh. Springerverlag, Ergänzunswerk, Berlin, IV/4, 1963, pp. 463-526.

16. Mariat F. : Notes épidémiologiques à propos des mycétomes. In Recent Progr. in Microbiol. Univ. Toronto press, Toronto, 1967, pp. 668-684.

17. Nicolau S. G., Avram A. : Micetomul cutanat. Ed. Acad. RSR., 1960.

18. Palmer P. E. S. : Kaposi's Haemangiosarcoma as seen in Central Africa. Dermatol. Tropica, 1963, 2, 67-78.

19. Segretain G., Mariat F.: Rceherches sur la présence d'agents de mycétomes dans le sol et sur les épineux du Sénégal et de la Mauritanie. Bull. Soc. Path. Exot. 1968, 61, 194-202.

20. Vanbreuseghem R. : The early diagnosis of Mycetoma. Derm. Internat. 1967, 6, 123-140.

21. Vanbreuseghem R., De Vroey Ch., Takashio M.: Guide to Medical and Veterinary Mycology, 2nd Ed., Masson, Publishing, 1978, New York, U.S.A.

22. Wesselinoff W.: Uber den histologischen Nachweiss von Vegetabilien in Fleischwaren. Deutsch. Tierärztl Wschr. Rundschau, 1943, 29/30, 1-7.

Adresse: Pr. Dr. med. V. A. Balabanoff, Dr. sci. Fr. Nansen 5, Sofia, Bulgarie. 